\title{
Good bugs, bad bugs: learning what we can from the microorganisms that colonize our bodies
}

he NIH recently announced the first recipients of funding for its Human Microbiome Project.

The number of microorganisms that live inside or on the body of a healthy adult human is thought to be least 10 times greater than the number of human somatic and germ cells (Figure 1). Some of these microorganisms are beneficial to the human host (e.g., some bacteria in the intestines break down carbohydrates that humans would otherwise not be able to digest), and some can cause illness (e.g., Streptococcus pneumoniae, which can be found in the nasopharynx of healthy individuals but causes disease if it colonizes other parts of the body, such as the lungs, where it causes pneumonia). However, the effects on human health of most of these microorganisms are not known; as Martin Blaser, Professor of Medicine and Microbiology at New York University School of Medicine, told the JCI, "We need to know who these microorganisms are and what they do." In an attempt to answer these questions and increase our understanding of how these microorganisms influence human health and disease, the NIH launched the Human Microbiome Project at the end of 2007 (1).

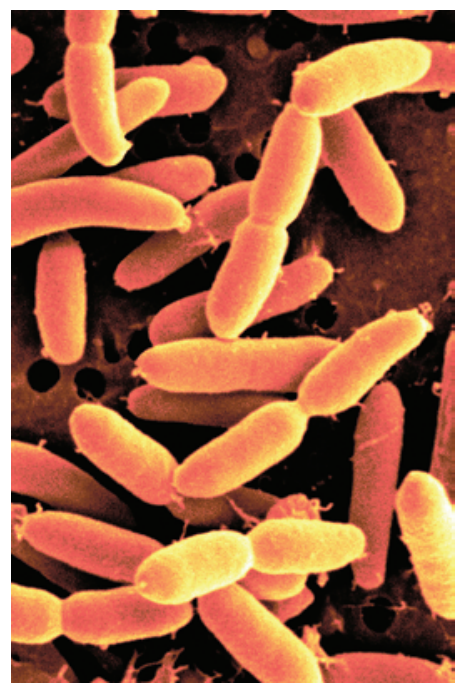

Figure 1

The genome of Lactobacillus acidophilus is part of the human microbiome, as $L$. acidophilus can be found in the mouth, intestines, and vagina. Image credit: Photo Researchers Inc.
The human microbiome consists of all the genomes of all the microorganisms that live inside or on the body of a healthy adult human. The idea behind the Human Microbiome Project is to use metagenomic and traditional genome sequencing approaches, which enable the analysis of all the DNA of all the microbes recovered in an environmental sample and of single clonal microbial cultures, respectively, to determine whether individuals share a core human microbiome and to understand whether changes in the human microbiome correlate in any way with changes in human health. Other goals of the project include developing new technological and bioinformatic tools to address the central issues and determining the ethical, legal, and social effects of research into the human microbiome.

The initial goal is to sequence 600 genomes from both cultured and uncultured bacteria as well as several nonbacterial microorganisms, focusing on the genomes of microorganisms in the digestive tract, mouth, skin, nose, and female urogenital tract. The aim of this goal is to provide researchers the background information to investigate the relationship between the microorganisms that contribute to the microbiome and human disease. Upon completion, the total number of microbial genomes sequenced will be raised to around 1,000, as some have already been completed, some are underway, and some will be sequenced as part of other projects that are already planned.

An estimated \$115 million will be distributed to researchers as part of the Human Microbiome Project over 5 years. Most of the recently announced initial round of funding, which will provide approximately $\$ 21.2$ million, is to support researchers developing innovative technologies - specifically technologies that should help improve and refine the way in which researchers identify the microorganisms that contribute to the microbiome - and computational tools. According to Alan Krensky, director of the Office of Portfolio Analysis and Strategic Initiatives, which oversees the NIH Roadmap for Medical Research, the NIH targeted initial funding in this area because "The development of new tools and technologies is central to our ability to meet the goals of the Human Microbiome Project. An exceptional amount of information will be generated by this project and we need robust technologies and analytical tools that are equal to the task" (2).

The remainder of the money awarded in this round of funding is going toward establishing the Human Microbiome Project Data Analysis and Coordination Center and to researchers examining ethical, legal, and social implications of human microbiome research. The Data Analysis and Coordination Center will act as a repository for all the data generated by the Human Microbiome Project, which will also be deposited in other public databases, including those supported by the National Center for Biotechnology Information (3).

The importance of the Human Microbiome Project was highlighted to the JCI by Blaser, who thinks that understanding how the microorganisms that contribute to the microbiome affect human health and disease is an area of research as exciting as stem cell research, with as much therapeutic potential. He continued that the microbiome is likely much more diverse than the human genome and that it changes over time as the microorganisms that colonize the human body change. By understanding these changes and taking a census of the microorganisms present under different conditions of health and disease, Blaser hopes that the Human Microbiome Project will lead to the identification of new drugs from microorganisms - just as drugs have been developed using botulinum toxin and cyclosporine A from the bacterium Clostridium botulinum and the fungus Tolypocladium inflatum Gams, respectively - and the use of approaches to manipulate the populating microorganisms to benefit human health; there is a "lot of potential" there, he says.

\section{Karen Honey}

1. NIH. Human Microbiome Project (HMP): overview. NIH Roadmap for Medical Research. http://www. nihroadmap.nih.gov/hmp/index.asp.

2. NIH. 2008 October 7. Human Microbiome Project awards funds for technology development, data analysis and ethical research [press release]. http:// www.eurekalert.org/pub_releases/2008-10/nhgrhmp100708.php.

3. NCBI. Entrez Map Viewer. http://www.ncbi.nlm. nih.gov/mapview. 